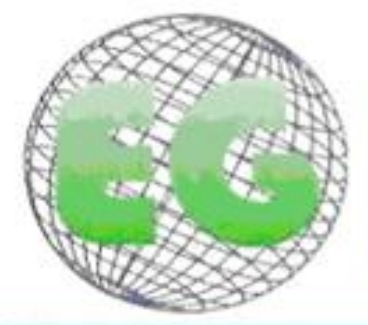

www.um.es/egloball

\title{
REVISIONES
}

\section{El cambio de turno: Un eje central del cuidado de enfermería}

The Handover: A Central Concept in Nursing Care

\section{*Guevara Lozano, Maryory **Arroyo Marles, Ligia Patricia}

*Magister en Enfermería. Profesora Facultad de Enfermería y Rehabilitación. Universidad de La Sabana. E-mailmaryorygl@unisabana.edu.co ${ }^{* *}$ Especialista en Gerencia en Instituciones de Salud. Directora Enfermería Clínica Universidad de La Sabana. Colombia.

\section{Palabras Clave: Cambio de turno; pase de guardia; cuidado de enfermería.}

Keywords: Shift change; Handover; Nursing care

\section{RESUMEN}

Con el propósito de reflexionar acerca del valor del cambio de turno como momento y eje central del cuidado de enfermería, se realizó una revisión de la literatura en idioma español, inglés y portugués, a partir de los descriptores: cambio de turno, informe de turno, pase de guardia, traspas de información, y comunicación (circunscrita a informes de enfermería). La búsqueda se hizo en las bases de datos SciELO, Ovid, Science Direct, Redalyc, Publindex, Lilacs, EBSCOhost Academic Search Premier, pubmed y Dialnet; se revisaron un total de 80 artículos de los cuales se seleccionaron 50 que contenían los criterios de inclusión.

Los resultados de la búsqueda evidenciaron gran cantidad de información frente al concepto, tipo, características y barreras, destacando la importancia que tiene para la disciplina comprender su significado en la práctica diaria.

En conclusión, se debe reflexionar sobre la importancia del permanente crecimiento profesional; los cambios los procesos y procedimientos pero aún más ratificar que el informe no es una oportunidad de un momento, es necesario educar, realizar seguimiento, evaluación y retroalimentación para permitir mejoras de los grupos y fomentar su práctica diaria. La revisión de literatura muestra una tendencia en estudios cualitativos y en menor proporción cuantitativos, además de escasa investigación en mejores prácticas, evaluación de las mismas y la eficacia de los protocolos o guías estructuradas, que contribuyan a través de la investigación a la práctica diaria al cuidado con calidad y calidez.

\section{ABSTRACT}

With the objective of reflecting on the value of the shift change as a central moment and concept in nursing care, a literature review was conducted in Spanish, English, and Portuguese using the following descriptors: cambio de turno [shift change], informe de turno [shift report], pase de guardia [handover], 
traspaso de información [information exchange], and communication (restricted to nursing reports). The search was conducted using the following databases: SciELO, Ovid, Science Direct, Redalyc, Publindex, Lilacs, EBSCOhost Academic Search Premier, Pubmed, and Dialnet; a total of 80 articles were reviewed, of which 50 were selected because they satisfied the inclusion criteria.

The search results showed a large quantity of information on concepts, types, characteristics, and barriers, emphasizing how important it is for the discipline to understand the significance of the handover in daily practice.

In conclusion, one should reflect on the importance of continued professional growth, changes, processes, and procedures; moreover, one should ensure that the shift report is not only a momentary but also a necessary opportunity to educate and perform monitoring, evaluation, and feedback to allow improvement of the groups and encourage daily practice. The literature review revealed a tendency toward qualitative studies and a lesser proportion of quantitative studies, in addition to limited investigations on best practices, their evaluation, and the efficacy of protocols and structured guidesall of which contribute to daily practice in warm, high-quality care.

\section{INTRODUCCIÓN}

La comunicación ha sido siempre un aspecto o elemento vital del proceso de cuidado de enfermería, especialmente durante la transferencia de información que con el devenir del tiempo y el avance científico y tecnológico ha traído consigo nuevos enfoques frente a la entrega de información con calidad y calidez. El cambio de turno o pase guardia es un momento de cuidado clave para garantizar la continuidad del cuidado y la seguridad de paciente, definido como un informe o ritual, dado en el momento en que el profesional de enfermería transfiere la responsabilidad de los pacientes y lo que ha realizado en su turno a otro que llega.

Con el propósito de reflexionar acerca del valor del cambio de turno como momento y eje central del cuidado de enfermería, frente a la seguridad del paciente y la continuidad del cuidado se realizó una revisión sistemática de la literatura. Este artículo presenta los resultados de la misma, frente a su concepto, características y una propuesta para optimizar el proceso e integrar al paciente y su familia como participantes activo del cuidado. Además de plantear una experiencia del informe en la Clínica Universidad de La Sabana.

\section{MÉTODO}

Se realizó una revisión de la literatura en idioma español, inglés y portugués, a partir de los descriptores: cambio de turno, informe de turno, pase de guardia, trasferencia de información, y comunicación (circunscrita a informes de enfermería). La búsqueda se hizo en las bases de datos SciELO, Ovid, Science Direct, Redalyc, Publindex, Lilacs, EBSCO-Fuente Académica, pubmed y Dialnet; así como una revisión manual en libros de fundamentación básica en Enfermería y dos protocolos de instituciones de salud de Colombia. Se revisaron un total de 80 artículos de los cuales se seleccionaron 50 que contenían los criterios de cambio de turno o pase de guardia como tema central, traspaso de información como comunicación en enfermería, cuidado de enfermería y propuestas de mejora. Las características de los artículos 
fueron: revisiones de temas, artículos reflexivos, investigativos, de carácter cualitativo y cuantitativo y protocolos o guías estructuradas. Se realizó una revisión final con los documentos escogidos, donde se identificó cómo se abordaba el tema de acuerdo con las palabras clave y el propósito planteado.

\section{RESULTADOS}

Del análisis cualitativo del concepto, las características y planes de mejora, frente al reporte o entrega de turno, por parte de los profesionales de enfermería en su práctica diaria, se plantean una tendencia por estudios cualitativos y en menor proporción cuantitativos, además de escasa investigación en mejores prácticas, evaluación de las mismas y la eficacia de los protocolos o guías estructuradas. Se reunieron 80 artículos de los cuales se tomaron 50 que reunieron los criterios de inclusión, de ellos el $70 \%$ (35) eran investigaciones cualitativos y el $30 \%$ (15) restante eran cuantitativos, publicados entre 1999 al 2013. Así mismo, se evidencio la escasa investigación en países de habla hispana, solo el $6 \%$ de los artículos fueron en español. El análisis de contenido arrojó la identificación del concepto, sus características, barreras en todos los artículos, la ética en tres, la importancia de la participación del paciente y la familia en 10 y de las estrategias de mejora en 20 que se describen a continuación:

\section{Concepto de Cambio de turno}

La revisión de la literatura muestra que el manejo del concepto es unificado y en muchos casos literalmente practicado, Nogueira ${ }^{(1)}$ lo define como los informes verbales que tienen como finalidad relatar lo ocurrido en la asistencia prestada a un paciente durante el turno anterior para que los integrantes del turno siguiente conozcan los aspectos fundamentales de su condición actual y garanticen la continuidad de la atención.

Hays $^{(2)}$ lo enuncia como la transferencia de información del personal saliente al personal entrante; se podría afirmar que el informe es una parte compleja e importante de la práctica profesional que atiende varios propósitos, incluyendo la comunicación, el suministro de información actualizada, la continuidad y la calidad del cuidado del paciente ${ }^{(3)}$, así como la asignación de las responsabilidades y tareas de rutina. Sexton lo define como un "proceso importante de intercambio de información para asegurar y mantener la continuidad y la calidad de la atención en pro de la seguridad del paciente, que cumple con las normas legales de la práctica profesional". Así mismo afirma que una de las dificultades que se presentan es que el informe carece a menudo de forma y estructura, por lo cual la información que se trasmite es irrelevante, repetitiva y especulativa, comprometiendo el proceso en varios casos por la falta de directrices ${ }^{(4)}$.

Los libros de fundamentos de enfermería lo refieren como un reporte vital para la continuidad del cuidado, en donde un grupo de enfermeras saliente puede reportar a todo el grupo entrante la información actualizada del paciente. El reporte debe ser completo y conciso sin importar el método empleado, enfocado en el paciente siguiendo un orden específico y no un momento de conversación social ${ }^{(5)}$. 
En el 2009, Terry lo definió como una responsabilidad y una rendición de cuentas del cuidado de un paciente, que se transfiere de una enfermera a otra, donde la comunicación está vinculada a la seguridad del paciente y a la continuidad de su cuidado $^{(6)}$. En su estudio encontró que las fallas en este proceso interferían en la continuidad del cuidado, y los diferentes espacios para realizar el informe y su tiempo, los cuales varían de una institución a otra.

La importancia que tiene el cambio de turno, en el proceso de atención de los pacientes ha motivado el desarrollo de protocolos para estandarizar el proceso en las diferentes instituciones de salud, sin embargo, la literatura muestra que los resultados obtenidos no son satisfactorios ${ }^{(7),(8)}$. Justamente, en la mayoría de instituciones de salud, existen protocolos o guías de cambio de turno y lo definen como una actividad que garantiza la continuidad del cuidado del paciente por parte del personal de enfermería, al igual que la asistencia de elementos necesarios para su atención, como el inventario del servicio o informe de alguna novedad ocurrida con los mismos ${ }^{(9)}$. Al mismo tiempo, de forma más explícita, se describe como un procedimiento por medio del cual se recibe el informe de cada uno de los pacientes incluyendo: diagnostico, evolución, tratamiento, actividades y exámenes realizados o pendientes ${ }^{(10)}$.

Un informe planteado como un método para proporcionar información relevante para orientar la práctica diaria y el cuidado directo. El reporte, por tanto, debe ser, informativo y educativo para las enfermeras, los pacientes y sus familias, donde se compartan detalles no solo del estado del paciente y su tratamiento, conocido o desconocido por ellos mismos, sino la planificación del cuidado.

\section{Características del cambio de turno}

\section{Periodicidad, frecuencia, duración, lugar, participantes y tipo de información.}

El cambio de turno se caracteriza porque es un evento rutinario, diario que se lleva a cabo en la mañana, tarde y noche ${ }^{(11)}$. Con un promedio de tiempo determinado para su desarrollo de 15 a 45 minutos, que varía según el número de pacientes y su condición, este es uno de los retos más reafirmados en los profesionales de enfermería para mantenerse dentro del marco de tiempo asignado ${ }^{(4)}$. Athwal et al. y Hays han abordado principalmente el tipo, la duración, el contenido o características, sus ventajas y desventajas ${ }^{(2,12)}$, así como, pruebas con cambios en el proceso frente al paciente y su familia ${ }^{(13)}$. Riesenberg et al. hacen hincapié en la complejidad del informe y las dificultades, como la falta de comunicación y la trasferencia inadecuada de los cuidados ${ }^{(14)}$.

En él, se comparte información sobre aspectos físicos, psicosociales y espirituales de los pacientes, así como hechos, opiniones e impresiones médicas, para resolver problemas de cuidado, dar y recibir apoyo, construir relaciones y gestionar conflictos. El informe debe ser lo suficientemente amplio como para abarcar el carácter holístico, y objetivo del cuidado a largo plazo del paciente, pero lo suficientemente específico para satisfacer metas a corto plazo y ofrecer las preferencias individuales para 
satisfacer las necesidades de los pacientes y no los de los profesionales de enfermería.

Por ello, el cambio de turno significa un tiempo de comunicación para promover el cuidado, la seguridad y las mejores prácticas ${ }^{(15)}$, encaminadas a eliminar o mitigar los riesgos para el paciente ${ }^{(16)}$. Además el significado personal y la socialización del mismo, puede ser el propósito y voluntad personal para recibir a los pacientes y sus familias durante la entrega, puesto que el informe puede tener un significado emocional $^{(6)}$. Un ejemplo de ello es, la participación en las dificultades o angustias por parte de las enfermeras en este momento de cuidado, entendido como una ocasión para el mismo, un momento o espacios de interrelación del personal de enfermería con el paciente y su familia, en los que se genera, se da y se afianza el cuidado de enfermería ${ }^{(17)}$.

Así, el cambio de turno se convierte en ese momento, para conectarse con el paciente, su familia y el resto del personal, para resolver con el equipo completo las dificultades tenidas en el transcurso de su turno, y así mismo socializar, compartir acerca de cómo se resolvió y lo que está pendiente. Lo importante es que no sea una rutina que transponga los tiempos para el desarrollo del proceso, son personas cuidando a otras personas, por esto no se puede desconocer esta voluntad personal.

Igualmente, los lugares donde se lleva a cabo el informe son las estaciones de enfermería, sala de conferencias, cabecera del paciente, o pasillo ${ }^{(18)}$. Las formas más comunes son el reporte oral y el escrito, le sigue el grabado, que tiene una desventaja y es la no retroalimentación ${ }^{(5)}$ y por ultimo con el paciente, en este él es incluido y se entera del informe dado a la enfermera entrante, la literatura coincide que el mejor lugar para realizar el informe, es este, con el paciente y especialmente en el turno de la noche.

\section{Barreras en la transferencia de la información}

En 2010 Riesenberg et al $^{(14)}$ realizaron una revisión sistemática de la literatura que identificó las barreras para la adecuada transferencia de información y las clasificaron en ocho categorías: barreras de comunicación, problemas en la estandarización o políticas que aseguren el proceso, dificultades en los equipos tecnológicos, factores del entorno, disponibilidad de tiempo, dificultades por la complejidad y el número de pacientes, entrenamiento y educación del personal y los factores humanos. A pesar de las conocidas consecuencias negativas de transferencias inadecuadas de información, muy poca investigación se ha hecho para identificar mejores prácticas. Existe una notable coherencia en las estrategias sugeridas, pero hay una falta de evidencia para apoyarlas ${ }^{(19)}$.

Los métodos tradicionales tienden a ser largos, incoherentes e incorrectos en la información del paciente, el contenido a veces se desvía a declaraciones irrelevantes o de juicio a priori que conducen a perjuicios negativos de los profesionales hacia los pacientes ${ }^{(20)}$. Puede así convertirse el informe en un vehículo para el irrespeto, la falta 
de comunicación, la negatividad inconsciente, las proyecciones o hechos no verificados, que desmejoran las relaciones y los valores éticos ${ }^{(21)}$.

Otro factor que influye en el recibo y entrega de turno son los distractores como las conversaciones paralelas durante la entrega de turno, el movimiento de carros de suministros, ropa y alimentos, máquinas de limpieza, volumen alto de la radio y la televisión, llamadas telefónicas, interrupciones por parte del personal médico y de apoyo, conversaciones en el corredor y en la central de enfermería, situaciones que son más frecuentes durante los turnos de la mañana y tarde por ser el horario de mayor circulación ${ }^{(22)}$.

La información incompleta, los distractores y los errores en la comunicación ${ }^{(23)}$ durante el recibo y entrega de turno, son la principal causa de eventos centinela, tales como errores de medicación, estancias hospitalarias más prolongadas toma de pruebas de diagnóstico innecesarias, e insatisfacción del paciente ${ }^{(24)}$. Este contexto provoca conductas del personal, que no contribuyen al buen proceso y se convierte en un círculo vicioso que incide en la misma entrega de turno, puesto que generan respuestas negativas, que no contribuyen en la continuidad y el juicio crítico de los profesionales para priorizar y organizar su cuidado, ejemplo de ello, es la información no entendida o la omisión de la misma, la prolongación del tiempo de entrega, la aglomeración e indisciplina por parte del equipo de enfermeras y auxiliares, desviando la atención, mostrando desinterés, desmotivación, falta de respeto entre colegas e interrupción en comunicación ${ }^{(1)}$

Los horarios de trabajo de las enfermeras, constituyen otro factor predisponente en la eficacia y eficiencia de la entrega de turno, tomando como referencia los horarios de inicio y finalización de trabajo, en muchos casos existe superposición de apenas 10 minutos entre los turnos de la mañana-tarde y los turnos de tarde-noche. Evans et al. afirman que la ausencia o insuficiencia de tiempo disponible para este proceso, afecta la comunicación en la entrega de turno ${ }^{(25)}$. Sugieren que los mismos pueden influenciar negativamente en el desempeño del grupo de enfermería, como emisor de la información y en consecuencia, se desarrollan implicaciones tanto para la asistencia como para la gestión del servicio.

\section{La ética en la entrega de turno}

Hablar de la ética de este momento de cuidado, es un punto importante y frecuentemente referenciado en la literatura ${ }^{(26,27)}$, justamente, el compromiso principal de las enfermeras frente al cuidado implica equilibrar los beneficios y las cargas de compartir la información a la que se tiene acceso con otros. Como defensores de intimidad los pacientes y profesionales colegas, los profesionales de enfermería deben ser claros acerca de sus intenciones para compartir hechos y opiniones, y así determinar cuál será el manejo de la información confidencial relevante para el cuidado del paciente, que implica juicios de valor sobre lo que es importante para su cuidado y lo que está autorizado para compartir y bajo qué circunstancias. Del mismo 
modo, discernir qué información va a divulgar dentro del contexto de relaciones colegiales y qué se requiere para el trabajo en equipo ${ }^{(21)}$.

Los profesionales de enfermería al manejar información y tomar decisiones, deben basarse sobre principios asociados a la justicia, el respeto, la dignidad y el compromiso, y no las necesidades propias o sociales. Justamente el razonamiento moral cambia con el tiempo, gracias al nivel de razonamiento, resultado de la madurez cognitiva, la experiencia en su práctica, la educación formal, y el medio ambiente $^{(28)}$. Además se espera que se adhieran a los valores de los códigos éticos de la práctica profesional, esquema de principios que demuestran la responsabilidad del los miembros de la profesión a la sociedad. El Código de Ética ${ }^{(29)}$ para Colombia resume los valores y derechos de las enfermeras a seguir para tomar decisiones éticas y proporcionar cuidados de enfermería de alta calidad.

Con esta concepción, el informe debe verse como un vehículo para compartir valores, creencias, percepciones, juicios y sobre todo respeto. Cuando se establece un cambio de turno de manera fundamentada, en la ética y responsabilidad, la calidad y la seguridad del cuidado, mejoran las relaciones. se fortalecen la eficiencia y el funcionamiento del equipo de trabajo, se optimizan los tiempos y no se da información equivocada ni insuficiente.

\section{Importancia de la participación del paciente y la familia}

Evidentemente, el proceso no ha incluido tradicionalmente a los pacientes y sus familias $\mathrm{y}$, de hecho, las familias son a menudo requeridas para que se retiren de la habitación durante el proceso ${ }^{(6)}$. En estudios realizados entre el 2006 y $2007^{(30),(31)}$ se mostró que los profesionales de enfermería ven como un problema serio realizar el informe con los pacientes, puesto que podría alargar en lugar de acortar los tiempos, con ocasión de las preguntas realizadas y la interacción con ellos mismos o su equipo, lo que resultaría en largos informes.

De otro lado, los profesionales de enfermería no advierten que los mismos pacientes y sus familias son administradores de su propio cuidado en la estancia hospitalaria, no se les da una oportunidad de escuchar y participar en el intercambio de información ${ }^{(22,32)}$. Igualmente, la rendición de información entre los turnos se promueve mediante la observación directa del paciente por ambas enfermeras y no en supuestos, cuando el informe no se realiza con el paciente, se pierde la oportunidad de visualizarlo, -incluyendo su familia- para planificar el cuidado.

Sin embargo, mientras que muchas enfermeras ya reconocen el valor de entregar el informe cara a cara con el paciente y lo practican de manera rutinaria aún sigue siendo relativamente poco común. A pesar de los desafíos en la implementación del informe con el paciente, existen investigaciones que demuestran los beneficios de este proceso ${ }^{(22),(30),(33)}$. Uno de ellos, es la capacidad de la enfermera para confirmar inmediatamente la información con la visualización del paciente y obtener una evaluación de referencia para comparar frente a los cambios durante el turno ${ }^{(34)}$, planificando y priorizando los cuidados al paciente. 
La evidencia sugiere que los pacientes mejor informados son menos ansiosos y más adherentes en sus cuidados, más autónomos en sus tratamientos y metas y se sienten satisfechos ${ }^{(27),(35)}$. Además, los costos de salud bajan por la autogestión por parte de los pacientes y el uso más eficiente de los recursos. Los beneficios de realizar la entrega con el paciente incluyen no solo la visualización de los pacientes y el cuidado prioritario e integral, sino mayor satisfacción del profesional, que alienta el trabajo en equipo de todos los turnos, promueve la responsabilidad personal y compromiso de todos ${ }^{(30)}$.

Cabe aclarar, que la comunicación de la información es un derecho para el paciente y su familia ${ }^{(36)}$, y se reglamenta sobre la Ley 911 de 2004, que orienta el proceso de cuidado ${ }^{(29)}$, asegurando una visión unitaria que busque atender sus dimensiones físicas, sociales, mentales y espirituales, para brindar un cuidado de enfermería que permita comprender el entorno y las necesidades de los pacientes con respeto cultural y dignidad de la persona sin discriminación; garantizando un diálogo que fundamente la interrelación enfermera - paciente y familia, como elemento esencial del proceso del cuidado, que asegura una comunicación efectiva, respetuosa, basada en relaciones interpersonales armoniosas, participativas en el cual el paciente y la familia expresan con libertad y confianza sus necesidades y expectativas de cuidado $^{(37)}$.

Al respecto son varios los artículos que soportan la búsqueda real de la atención centrada en las necesidades y expectativas del paciente y la familia ${ }^{(38)}$ a fin de generar el desarrollo de una cultura de la educación inclusiva, solidaria, que reconozca y permita la participación de los pacientes en la actualidad, que están acostumbrados a un cierto nivel de servicio y un alto grado de poder para decidir lo que quieren y cómo quieren. A diferencia de sus más pasivos predecesores, los pacientes modernos piden las interacciones de atención pues cuentan con una gran cantidad de información. Por otra parte, no están dispuestos a aceptar el "médico sabe mejor" enfoque del pasado. En general, pacientes ( $y$ tal vez más importante, a sus familias) son cada vez más vocales y exigentes con la calidad de cuidado que se ofrece en las instituciones de salud.

Otro beneficio incluye la interacción, promoviendo la relación enfermera-paciente ${ }^{(30)}$. Como resultado, el personal trabaja como un equipo y mejora la imagen profesional de la enfermera. Al trabajar juntos, los pacientes son testigos de una transferencia segura y profesional de las responsabilidades. Los pacientes pueden hacer preguntas o aportar información al debate. Permitir que el profesional y el paciente tengan la oportunidad de compartir la información, promueve la participación y mejora la satisfacción $^{(33)}$. Construye relaciones entre los miembros del personal, demostrando un cuidado centrado en el paciente y no en los medios para llegar a él.

Así, pacientes y familias tienen comunicación de un testimonio personal, del profesionalismo de la enfermera y la organización de salud. Incide en la recuperación y aclaración de la información errónea que se comparte durante el informe, además de la educación para los pacientes y familias al recibir nueva información y es la 
oportunidad personal de tomar un modelo a seguir por parte de otra enfermera que pueda tener menos experiencia.

Romper la rutina tradicional del informe es un reto para los profesionales de enfermería, pues en definitiva, no se contempla, que los pacientes y sus familias, sean garantes de una atención segura y de la experiencia de cuidado. La reflexión que se quiere enfatizar en este artículo es que este proceso de informe podrá ser nuevo y desafiante, buscando optimizar la continuidad del cuidado, fortaleciendo la relación enfermera-paciente y el trabajo del grupo de salud.

\section{Estrategias para mejorar el recibo y entrega de turno}

Se proponen, con base en la literatura, las siguientes estrategias que pueden ser útiles en la optimización del proceso a partir de las barreras que se presentaron anteriormente:

- Perspectiva de enfermería; es importante definir un concepto de cuidado y una visión que se relacione con la misión y visión de la institución que fundamente la práctica diaria en este momento de cuidado, discutida en un consenso con todos los profesionales de las unidades o dependencias, que reconozca la importancia del acceso de los pacientes y familiares a la información en la entrega de turno. Esta visión de cuidado de la organización de salud, debe contener la relación entre los resultados de cuidado, la satisfacción del paciente y la familia, así como la participación en el proceso de atención de enfermería y la toma de decisiones ${ }^{(39)}$.

- Preservar la intimidad y confidencialidad; en primera instancia se requiere un cambio en la práctica profesional, que puede llevar también a un ajuste paradigmático, a causa de sus características y desventajas, en tiempos, espacios, personal y uno de los que más se refiere en la literatura, la intimidad en el manejo confidencial de la información. La información confidencial es divulgada inadvertidamente durante todo el proceso de hospitalización, por parte de todo el equipo de salud, estas revelaciones no son intencionales, sino consecuencia de tener espacios comunes. De hecho, la forma para limitar esto, es durante el mismo proceso de entrega de turno con la conciencia de que se está con el paciente, planeando con anticipación el tipo de información nueva o sensible que pueda ser compartida antes o después de estar con el o si amerita hablarlo luego en privado.

- Participación del paciente y la familia; los espacios en muchos casos son compartidos y esto deriva en la preocupación de los profesionales frente al desarrollo del informe con los pacientes y familiares, pero esto no debe impedir oírlos, particularmente para planificar su cuidado, tomar decisiones y resolver las necesidades que contribuyan con la continuidad del cuidado y la atención segura del paciente. En la admisión, se puede preguntar si quiere participar o no del proceso con un familiar o el mismo paciente. Para facilitar la participación del paciente y la familia, optimizar el tiempo y preservar la intimidad e individualidad, se recomienda realizar un informe general de los pacientes de 5-10 minutos en el comienzo de cada turno en la estación de enfermeras o en una sala de 
conferencias con el personal de enfermería reduce las dificultades en el manejo de la información confidencial.

Terry $^{(6)}$ plantea que se brinde una cantidad limitada de información como son los diagnósticos básicos, las cuestiones de seguridad y las cuestiones de cuidado que todos deben saber. Después de esto dar un informe con el paciente en las habitaciones y compartir lo que ha ocurrido durante el turno, el plan de cuidado y la bienvenida al paciente y su familia a participar en la discusión. Durante el informe, hay una comprobación de seguridad de fluidos, vía intravenosa, drenajes, medicamentos que estén en infusiones continuas, y todo lo referente la medición de escalas para la seguridad del paciente e inventarios, los cuales pueden ser delegados a su grupo de trabajo, para que los profesionales se concentren en las necesidades de cuidado de los pacientes.

Para el manejo e inclusión de los pacientes Douglas y Cairns (40),(41), proponen realizar los informes en la noche e involucran tres pasos; el primero es involucrar al paciente y la preferencia de tener miembros de la familia o no durante el informe, además de fomentar la confianza del paciente por gestionar su cuidado. En segundo lugar, discutir temas de su cuidado como actualización clínica, por ejemplo, resultados de laboratorio, cuidado de heridas y medicamentos, discutiendo las metas de atención para el próximo turno. Por último, facilitar la comunicación bidireccional. Igualmente, como cualquier proceso nuevo, se debe dotar de educación a los profesionales de enfermería y a los pacientes en habilidades de comunicación, esta educación y apoyo del personal y los pacientes deben ser permanentes.

Para el tiempo requerido en el informe con la participación de los pacientes, se plantean negociaciones para satisfacer las necesidades de los mismos y sus familias en el mismo proceso, una opción es tomar nota del comentario y volver luego del informe para aclarar o satisfacer las necesidades y siempre pedir su aceptación. Otro potencial adicional en el ahorro de tiempo, se da cuando el paciente y la familia se entrevistan con las dos enfermeras ${ }^{(6)}$. De hecho, los profesionales deben concentrarse en el paciente y el informe y no en problemas circunscritos que él acarrea, que podrían ser manejados por enfermerías coordinadoras del servicio.

- Normalización; la normalización es otra estrategia que influye positivamente en el proceso, el uso de bitácoras o formatos estandarizados ${ }^{(12)}$ para la información tiene un uso frecuente y facilita o disminuye la posible variabilidad en la comunicación del reporte. Así mismo, se puede trabajar con los pacientes y las familias para determinar qué otros aspectos del cuidado deben ser incluidos en la plantilla del informe. Cuando estos formatos se desarrollan, los temas no técnicos también deben ser incluidos, un ejemplo de ello, es la planeación de visitas especiales o que el paciente espere, artículos que sean necesarios para su cuidado y que no estén en la institución o aspectos de trámites administrativos. 
- Disponibilidad de recursos; en cuanto a la disponibilidad de recursos físicos, estos deben estar fácilmente disponibles de una manera similar a cuando se introduce un nuevo equipo. Para mejorar el sistema de documentación se sugiere el uso de la tecnología informática y los modos de comunicación inalámbricos ${ }^{(42)}$, esto se explora como un medio para mejorar el proceso de cambio de turno y, posteriormente, los resultados de salud y seguridad del paciente ${ }^{(34)}$. El uso de la tecnología como sistemas móviles Android® o tablas permitirá el mejor registro y actualización segura de la información y prevenir errores que contribuyan a incidentes o eventos adversos.

\section{Análisis de una experiencia - Modelo de Cuidado de Enfermería Clínica Universidad de La Sabana}

Del análisis de las anteriores consideraciones relacionadas con el cambio de turno, con enfoque de gestión de cuidado, mejora continua y niveles superiores de calidad, surgen desde la práctica de enfermería para la Dirección de Enfermería en la Clínica Universidad de La Sabana varios interrogantes: ¿Cómo consolidar más allá de la rutina el cambio de turno como momento de cuidado para la adaptación de la persona frente a su constante salud-enfermedad, a la práctica diaria de enfermería? ¿Cómo incorporar en dicha práctica el uso de la tecnología para facilitar el manejo eficiente del tiempo, el seguimiento de los planes de cuidado, las intervenciones de enfermería, el control, seguimiento y prevención de los riesgos y la síntesis de las experiencias de los pacientes? ¿Cómo crear la necesidad de la responsabilidad compartida por enfermería para hacer de la entrega de turno un rito, una función enriquecedora de la práctica de enfermería? Y por último, ¿cómo lograr la participación de los pacientes y las familias en la presentación y la continuidad de información y experiencias vividas con la participación del equipo de salud tratante guardando y preservando el principio de confidencialidad?

Para dar respuesta a estas y otras inquietudes de gestión de cuidado se inicia desde el 2012 una propuesta de Modelo de Cuidado de Enfermería propio de la Clínica Universidad de La Sabana en Chía, Colombia, que parte de la visión ontológica y el concepto epistemiológico de cuidado de enfermería (elaborado por docentes de la Universidad de La Sabana y enfermeras de la Clínica ), propuesta que incluye entre otros el concepto de entrega de turno como momento de cuidado de enfermería en la Clínica y su optimización.

\section{Enfoque teórico del cuidado de enfermería en la Clínica Universidad de La Sabana en el cambio de turno.}

El cuidado de enfermería, debe ser provisto de una manera integral y con una visión de reciprocidad ${ }^{(43)}$, donde se conciba a las personas como seres cambiantes que enfrentan diariamente situaciones que afectan o mejoran su constante de salud enfermedad, es decir un proceso de conciliación permanente y activo, donde el profesional debe ser consiente de proveer un cuidado para la adaptación, una respuesta intencional, holística e interpersonal que se desarrolla mediante procesos 
cognitivos y habilidades profesionales que buscan como resultado promover la adaptación de la persona ${ }^{(44)}$.

Cuidar en si implica un espacio y un momento, además de un conocimiento propio de cada paciente, en actitudes, aptitudes y motivaciones, además, de una relación recíproca, única y auténtica, entre el profesional de enfermería y paciente, capaz de generar confianza, seguridad y apoyo efectivo. Lo anterior destaca la importancia de la trasferencia de información o cambio de turno, como momento de cuidado, donde se vivencia su concepto, como parte fundamental de la práctica diaria con un enfoque teórico disciplinar.

Con base en este concepto de cuidado, se elaboró la propuesta docente asistencial, entre la Facultad de Enfermería y Rehabilitación y la Dirección de Enfermería de la Clínica Universidad de La Sabana, que buscaba dar consistencia a la exploración de estrategias para incorporar en el trabajo diario de enfermería el eje de gestión de tecnología, como uno de los cuatro ejes del modelo evaluativo del sistema único de acreditación en salud, proceso que había iniciado la Clínica.

Se consideró la entrega y recibo de turno como momento de cuidado y punto de partida inicial del proyecto donde se hace evidente la reunión de todo un equipo humano compuesto por personas calificadas, comprometidas con un propósito en común, no sólo para proveer información relacionada con el paciente sino para evaluar y garantizar la eficacia del servicio en términos de cumplimiento de metas y objetivos propuestos, eficiencia en términos de la mejor utilización de los recursos y calidad en el logro de la satisfacción de necesidades y expectativas del paciente de turno mediante la implementación de la bitácora, la planeación de cuidados de enfermería, la continuidad y evaluación con el empleo del sistema HOSVITAL (plataforma de información de la Clínica). Se seleccionó el área de Hospitalización para la realización del proyecto por las características del proceso de atención; las condiciones clínicas de los pacientes que ingresan y la aplicación del modelo de cuidado de enfermería propuesto.

El proyecto de gestión se realizó en tres fases:

Una primera fase de Planeación y Diseño de la normalización y estandarización de la información: se realizó un proyecto de gestión liderado por docente, directora y coordinadora de enfermería de la Clínica, adelantado por estudiante de VIII semestre de la Facultad de Enfermería y un grupo focal de enfermeras del servicio de Hospitalización que compartían inquietudes sobre el tema, con una motivación particular en abordar el conocimiento empírico y teórico de enfermería; en aplicar en un contexto práctico los patrones del conocimiento de enfermería para enriquecer la práctica desde la teoría. Es necesario anotar que este grupo focal tenía como característica la reciente vinculación a la Institución, apertura y disposición a aportar, aprender y mejorar sin restricción con los cambios propuestos; su condición de profesionales dispuestas, líderes con enfoque a la mejora, la innovación y al cambio, con capacidad crítica frente a los hallazgos, determinó su participación. 
En esta fase se realizó un análisis de la perspectiva de enfermería con todo el grupo y paralelamente se revisó la literatura y se elaboró un primer instrumento: una bitácora manual de 16 ítems que comprendía los siguientes aspectos: identificación del paciente, datos subjetivos (¿cómo está?, ¿cómo se siente?), diagnóstico de enfermería, diagnóstico médico, valoración de riesgos (lesión de piel, caídas, alergias), accesos venosos, control de signos vitales, dispositivos respiratorios, dieta, dispositivos de nutrición, valoración de piel (heridas, zonas de presión, úlceras), dispositivos de eliminación, laboratorios, plan de cuidados, interconsultas, pendientes.

Se realizaron observaciones, relacionadas con el tiempo invertido para el recibo y entrega de turno; la identificación de oportunidades de mejora para precisar la información relacionada con paraclínicos, inicio de tratamientos farmacológicos, cambios en esquemas terapéuticos, continuidad al plan de atención y al proceso de cuidado de enfermería, identificación de posibles riesgos de eventos e incidentes relacionados con la falta de información y necesidad de hacer énfasis en la valoración del dolor y la identificación de alergias en la valoración del paciente.

En la segunda fase se realizó la validez facial de la bitácora de 16 ítems. Con el grupo focal, mediante la revisión de 12 bitácoras de enfermería entrega y recibo de turno del servicio de hospitalización escogidas al azar, de cada uno de los diferentes turnos de los meses de septiembre y octubre de 2012, se analizaron 119 registros en los que se encontraron los siguientes datos: Es evidente que no se tiene claridad de los datos que se deben llenar en la bitácora, El no registro de signos vitales por parte del profesional de enfermería, Se puede observar también que de 119 pacientes, solo 51 pacientes requerían valoración del dolor de los cuales se realizaron 9 pero no empleo la escala numérica para su valoración, mientras 42 pacientes que requerían valoración no fue realizada.

Se evidencia que los registros de diagnósticos de enfermería se están realizando en un $87 \%$ correspondiente a 104 registros, sin embargo, algunos de estos no cuentan con su correcta formulación. Mientras solo un 13\% correspondiente a 15 registros no se realizaron. Así mismo, se encontraron 104 registros de medicamentos en la bitácora, versus 15 registros que no se registraron y 111 registros de planes de cuidados de enfermería, sin embargo, muchos de estos solo indican asistencia en necesidades básicas y control de signos vitales. Solo 8 registros no se encontraron en las bitácoras.

En virtud de los hallazgos se realizaron las siguientes propuestas por parte del grupo foca: modificar el instrumento manual a digital con 24 ítems, incluyendo la especialidad tratante, la clasificación del aislamiento, identificación de alergias y valoración de Glasgow. Diseñar un instrumento para el personal auxiliar de enfermería en el que se incluya: registro de signos vitales, glucometrías, balance de líquidos, cambios de posición, cuantificación de drenajes y residuo gástrico. Dejar una sola casilla para el registro de la glucometrías de la entrega, organizar el instrumento de forma cefalocaudal, ya que en la entrega y recibo de turno se tienen dificultades o se omiten algunos datos. Eliminar la casilla de medicamentos, ya que muy pocas 
veces son actualizados ocasionando confusiones, además existen otros medios más eficaces como lo son las tarjetas de medicamentos y la entrega de carro de medicamentos y unificar el la casilla de cuidados de enfermería, los datos subjetivos y objetivos.

Igualmente, manifestaron dificultad para el registro de la bitácora por la no presencia del número de enfermeras asignadas al área (una enfermera por cada 12 a 14 pacientes); el tiempo que se emplea para su realización y la extensión en su contenido. Sin embargo, coinciden en expresar la ventaja del instrumento como el registro formal exigido por las instituciones y el marco legal para la entrega de turno y lo ven como el registro que les permite tener información actualizada y completa de la condición clínica, las necesidades de los pacientes y la priorización de los cuidados, según la valoración de dolor y de los riesgos.

Una tercera fase que consistió en la estandarización del instrumento en todo el servicio, la forma digital del mismo, la capacitación y el uso generalizado de las bitácora por parte de las doce enfermeras del servicio de Hospitalización de la Clínica; se propuso una discusión participativa con las enfermeras de cada uno de los turnos, para indagar sus puntos de vista acerca de la bitácora (fortalezas, aspectos a incluir, y aspectos a reevaluar). Así mismo, los medios para archivar estas bitácoras como: correo electrónico y bases en Excel y la implementación del instrumento para las auxiliares de enfermería por parte de la coordinación del servicio de hospitalización. Después de la capacitación y de tres semanas de aplicación, se verificó nuevamente encontrando: aumento significativo en el registro de los diagnósticos de enfermería, valoración de dolor, con escala análoga numérica, la actualización de laboratorios y planes de cuidado, con cambios en su planeación.

A la par, se observó que la planeación de los grupos focales para evaluar el contenido de la bitácora fue considerada como excelente o buena. Así como, la calidad de la bitácora en cuanto a creatividad del diseño, orden y contenido, refieren, igualmente, el crecimiento personal y profesional a través del uso del instrumento o bitácora. Que ratifica lo evidenciado en la literatura y parte de ellas mismas, desde su práctica diaria, en cuanto responsabilidad e importancia para la entrega de turno, que les permite resumir la historia clínica del paciente, llevar un seguimiento y registro completo e integral para dar continuidad a los cuidados de enfermería planteados, facilita la comprensión y mejora la entrega de turno.

En las sugerencias planteadas está capacitar y entrenar a las enfermeras recién vinculadas al servicio en el correcto uso de la bitácora y archivo de la misma para tener documento de recopilación de información. Revisar con las enfermeras en las jornadas de capacitación la aplicación de diagnósticos de enfermería, la evaluación de diligenciamiento de bitácoras de forma periódica y el posterior análisis de la información registrada para favorecer la aplicación de diagnósticos e intervenciones de enfermería, la identificación oportuna de indicios de atención insegura y la propuesta de barreras que minimicen los riesgos de la atención. 
Igualmente, Incorporar en la práctica de los grupos de enfermería la entrega de turno como un momento de cuidado con tiempos asignados y delimitados, contemplado como un rito o actividad grupal que no se debe interrumpir, a la que se debe prestar el mayor interés, preservando el principio de confidencialidad, la prudencia y el respeto, generando la participación de los pacientes y las familias.

\section{CONCLUSIONES}

En consenso y para finalizar, se debe reflexionar sobre la importancia del permanente crecimiento profesional, los cambios, los procesos y procedimientos pero aún más ratificar que el informe no es una oportunidad de un momento, es necesario educar, realizar seguimiento, evaluación y retroalimentación para permitir mejoras de los grupos y fomentar su práctica diaria. La revisión de literatura muestra una tendencia en estudios cualitativos y en menor proporción cuantitativos, además de escasa investigación en mejores prácticas, evaluación de las mismas y la eficacia de los protocolos o guías estructuradas, que contribuyan a través de la investigación a la práctica diaria al cuidado con calidad y calidez.

Los profesionales de enfermería como líderes del proceso deben ser capaces de guiar su realización siendo críticos y propositivos, con miras a eliminar los factores o barreras tanto personales como ambientales, que interfieren de modo negativo en el proceso, garantizando la continuidad, calidad y la seguridad del cuidado de los pacientes a través de una eficiente entrega de turno.

Un cambio de turno adecuado, aplicando su concepto, proporciona a los profesionales de enfermería la oportunidad de aclarar y corregir inexactitudes con el paciente y la familia, y con los mismos colegas, en última instancia, con la disminución de errores o eventos adversos en su cuidado y la satisfacción del paciente. Las observaciones y contribuciones por parte de ellos pueden ser valiosas para su cuidado, valorar el apoyo del paciente y la familia, en el intercambio de información es clave y así su cuidado trascienda en el tiempo. Por lo tanto, ellos deben ser incluidos en el proceso.

\section{Recomendaciones}

Basado en la revisión de la literatura de cambio de turno en enfermería y la experiencia empírica desarrollada en el 2012, se recomienda que, en el futuro, se realicen investigaciones cuantitativas que proporcionen evidencia para apoyar el uso de una estructura específica, bitácora, protocolo o método para la mejora del informe. Se necesitan con urgencia resultados de estudios de alta calidad centrados en los factores como; eficacia de los protocolos y las intervenciones de mejora y la participación del paciente y la familia. Así mismo, optimizar el informe con las estrategias mencionadas, en particular la participación del paciente y su familia en la práctica diaria con calidad y calidez. 


\section{REFERENCIAS}

(1) Nogueira MS, Mendes IAC, Trevizan MA, Fávero N, Hayashida M. Entrega de Turno Análisis de Factores en la Comunicación. Horizonte de Enfermeria 1999;10(1):8-18.

(2) Hays MM, Dsn,. The Phenomenal Shift Report: A Paradox. Journal for Nurses in Staff Development - JNSD 2003 January/February;19(1):25-33.

(3) Meissner A, Hasselhorn H, Estryn-Behar M, Nézet O, Pokorski J, Gould D. Nurses' perception of shift handovers in Europe: results from the European Nurses' Early Exit Study. J Adv Nurs 2007;57(5):535-543.

(4) Sexton A, Chan C, Elliott M, Stuart J, Jayasuriya R, Crookes P. Nursing handovers: do we really need them? J Nurs Manag 2004;12(1):37.

(5) White L, Duncan G, Baumle W editors. Foundations of Basic Nursing $<b r />$. Third Edition ed. USA NY: Delmar Cengage Learning; 2011.

(6) Griffin T. Bringing change-of-shift report to the bedside: a patient- and familycentered approach. J Perinat Neonatal Nurs 2010 April;24(4):348-353.

(7) Jukkala AMCNL, C.N.E., James DDNP, M.S.N., Autrey PMSN, Azuero A, Miltner RRNC. Developing a Standardized Tool to Improve Nurse Communication During Shift Report. J Nurs Care Qual 2012 July/September;27(3):240-246.

(8) Klee KMSN,B.C., Latta LNEABC, DavisKirsch SC, Pecchia MMSN, A.R.N.P. Using Continuous Process Improvement Methodology to Standardize Nursing Handoff Communication. J Pediatr Nurs 2012 April;27(2):168-173.

(9) Dirección Enfermería. Guía de entrega y recibo de turno. 2012; Available at: http://portalservicios.unisabana.edu.co/doc/ClinicaUniversitaria/gestionCalidad/Pagina s/Guias.aspx. Accessed 06/13, 2013.

(10) Martínez L, Sabogal P, Rojas R, Plazas M. Guía de recibo y entrega de turno. 2011; Available

at:

http://www.esevictoria.gov.co/sitio2/Guias_Protocolos/ENFERMERIA\%20/RECIBO\%2 OY\%20ENTREGA\%20DE\%20TURNO.pdf. Accessed Mayo, 2012.

(11) Skaalvik MW, Normann HK, Henriksen N. To what extent does the oral shift report stimulate learning among nursing students? A qualitative study ${ }^{*}$. J Clin Nurs 2010 Octobre;19(15-16):2300-2308.

(12) Athwal PMSN, Fields WDNS, F.H.I.M.S.S., Wagnell EBSN. Standardization of Change-of-Shift Report. J Nurs Care Qual 2009 April/June;24(2):143-147.

(13) Taylor C. Assessing patients' needs: does the same information guide expert and novice nurses? Int Nurs Rev 2002;49(1):11-19.

(14) Riesenberg LA, Leitzsch J, Cunningham JM. Nursing handoffs: a systematic review of the literature. Am J Nurs 2010 April;110(4):24-34.

(15) Spanke MT, Thomas T. Nursing assistant walking report at change of shift. J Nurs Care Qual 2010 Jul-Sep;25(3):261-265.

(16) Joint Commission on Accreditation of Health Care Organization (JCAHO). Sentinel event alert: Fatal falls. 2000; Available at: www.jointcommission.org/SentinelEventAlert/sea_14.htm. Accessed Mayo/15, 2013.

(17) Dirección Enfermería. Modelo de Gestión de Enfermería. Clïnica Universidad de La Sabana 2012 Agosto;1(1):1-42.

(18) Siqueira, Ivana Lucia Correa Pimentel de, Kurcgant P. Shift report: talking about paradigms and strategies . Acta Paul Enferm 2005 Abril;18(4):446-451. 
(19) Lawrence RH, Tomolo AM, Garlisi AP, Aron DC. Conceptualizing handover strategies at change of shift in the emergency department: a grounded theory study. BMC Health Services Research 2008 December;8:256-256.

(20) Elm J. Improving labor and delivery shift report. WHONN Lifelines 2004 Febrero/Marzo;8(1):54-59.

(21) Rushton $\mathrm{CH}$. Ethics of nursing shift report. AACN Advanced Critical Care 2010 Octubre/Diciembre;21(4):380-384.

(22) Hemant K, Zaheda J. Managing change in the nursing handover from traditional to bedside handover - a case study from Mauritius. BMC Nurs 2005;4(1):1-10.

(23) Chung K, Davis I, Moughrabi S, Gawlinski A. Use of an Evidence-Based Shift Report Tool to Improve Nurses' Communication. MEDSURG Nursing 2011 September/October;20(5):255-260,268.

(24) Blouin ASFACHE. Improving Hand-Off Communications: New Solutions for Nurses. J Nurs Care Qual 2011 April/June;26(2):97-100.

(25) Evans D, Grunawalt J, McClish D, Wood W, Friese CR. Bedside shift-to-shift nursing report: implementation and outcomes. Medsurg Nurs 2012 292; SepOct;21(5):281-284.

(26) Benner P, Tanner C, Chesla C. Expertise in Nursing Practice: Caring, Clinical Judgment and Ethics $<\mathrm{br} />$. Second Edition ed. United States of America: Springer Publishing Company; 2009.

(27) Meissner A, Hasselhorn H, Estryn-Behar M, Nézet O, Pokorski J, Gould D. Nurses' perception of shift handovers in Europe: results from the European Nurses' Early Exit Study. J Adv Nurs 2007;57(5):535-542.

(28) Ham K. Principled thinking: a comparison of nursing students and experienced nurses. J Contin Educ Nurs 2004;35(2):66-73.

(29) Congreso de Colombia. Ley 911 de 2004 . Por la cual se dictan disposiciones en materia de responsabilidad deontológica para el ejercicio de la profesión de Enfermería en Colombia. 2004; Available at: www.mineducacion.gov.co/1621/articles105034_archivo_pdf.pdf. Accessed Mayo, 2013.

(30) Anderson CD MR. Nurse shift report-who says you can't talk in front of the patient? Nurs Adm Q 2006;30(2):112-122.

(31) Caruso EM. The evolution of nurse-to-nurse bedside report on a medical-surgical cardiology unit. MedSurg Nursing 2007 Febrero;16(1):17-22.

(32) Ryan E, Steinmiller E. Modeling Family-Centered Pediatric Nursing Care: Strategies for Shift Report. Journal for Specialists in Pediatric Nursing 2004;9(4):123134.

(33) SandJecklin, Kari E, Sherman, Jay ME, Cnrn. Incorporating Bedside Report Into Nursing Handoff: Evaluation of Change in Practice. J Nurs Care Qual 2013 April/June;28(2):186-194.

(34) Strople B, Ottani P. Can Technology Improve Intershift Report? What the Research Reveals. Journal of Professional Nursing 2006 Mayo/Junio;22(3):197-204.

(35) Radtke K. Improving patient satisfaction with nursing communication using bedside shift report. Clin Nurse Spec 2013 Jan-Feb;27(1):19-25.

(36) Ministerio de Salud de Colombia. Resolución 13437, Por la cual se constituyen los comités de Ética Hospitalaria y se adoptan el Decálogo de los Derechos de los Pacientes. 1991 Noviembre.

(37) Congreso de Colombia. Ley 266 del 1996. Por la cual se reglamenta la profesión de enfermería en Colombia. 1996; Available at:

http://www.secretariasenado.gov.co/senado/basedoc/ley/1996/ley_0266_1996.html.

Accessed Mayo, 2013. 
(38) The Advisory Board Company. Elevating the Patient Experience Advancing Towards Person-Centred Care. 2012; Available at: http://www.advisory.com/. Accessed 04/15, 2013.

(39) Benson E, Rippin-Sisler C, Jabusch K, Keast S. Improving nursing shift-to-shift report. J Nurs Care Qual 2007 Jan-Mar;22(1):80-84.

(40) Wakefield D, Ragan R, Brandt J, Tregnago M. Making the transition to nursing bedside shift reports. Joint Commission Journal on Quality and Patient Safety 2012 June;38(6):243-1AP.

(41) Cairns LL, DNP D,Linda A., Hoffmann RL, Lorenz HL, Msn,. Utilizing Bedside Shift Report to Improve the Effectiveness of Shift Handoff. J Nurs Adm 2013 March;43(3):160-165.

(42) Wu MEIWEN, Lee TINGTING, Tsai TZUCHUAN, Lin KUANCHIA, Huang CHIYI, Mills MARYETTA. Evaluation of a Mobile Shift Report System on Nursing Documentation Quality. CIN: Computers, Informatics, Nursing 2013 February;31(2):85-93.

(43) Bueno Robles LS. Aspectos ontológicos y epistemológicos de las visiones de enfermería inmersas en el quehacer profesional. Ciencia y enfermería 2011 Abril;17(1):37-43.

(44) Moreno ME, Durán MM, Hernandez A. Nursing care for adaptation. Nurs Sci Q 2009;22(1):67-73.

Recibido: 9 junio 2013; Aceptado: 8 septiembre 2013

ISSN 1695-6141

() COPYRIGHT Servicio de Publicaciones - Universidad de Murcia 